\title{
GMR
}

\section{Molecular characterization, tissue distribution, and immune reaction expression of karyopherins in the domestic silkworm (Bombyx mori)}

\author{
J. Li ${ }^{1,2}$, L. Wang ${ }^{1}$, C. Qian' ${ }^{1}$, C.F. Zhang ${ }^{1}$, L.S. Dai ${ }^{1}$, Q.N. Liu', G.Q. Wei ${ }^{1}$, Y. Sun ${ }^{1}$, \\ D.R. Liu' ${ }^{1}$ B.J. Zhu ${ }^{1}$ and C.L. Liu ${ }^{1}$ \\ ${ }^{1}$ College of Life Sciences, Anhui Agricultural University, Hefei, China \\ ${ }^{2}$ College of Life Sciences, Huaibei Normal University, Huaibei, China \\ Corresponding authors: B.J. Zhu / C.L. Liu \\ E-mail: zhubaojian@ahau.edu.cn / cyschx@163.com \\ Genet. Mol. Res. 14 (4): 13049-13065 (2015) \\ Received May 29, 2015 \\ Accepted July 31, 2015 \\ Published October 26, 2015 \\ DOI http://dx.doi.org/10.4238/2015.October.26.1
}

\begin{abstract}
Karyopherins, including alpha and beta types, are transport proteins in the eukaryotic cell that carry cargoes across nuclear pore complexes into or out of the nucleus. In this study, full open reading frames of one beta and three alpha types of karyopherin were cloned from CDNA of the domestic silkworm (Bombyx mori). The one beta and three alpha types' open reading frames were $2661,1563,1515$, and 1551 base pairs long, respectively, and coded $886,520,504$, and 516 amino acids, respectively. The alphas all had one importin-beta-binding (IBB) domain, and eight, four, or seven armadillo/beta-catenin-like repeats. The beta had 19 HEAT repeat domains, which constructed one importin-beta- $\mathrm{N}$-terminal domain and one IBB domain. The recombinant proteins were expressed in Escherichia coli cells. The molecular weight of the beta type was approximately 100 $\mathrm{kDa}$, and the alphas weighed approximately $60 \mathrm{kDa}$. Phylogenic tree construction revealed that the alphas could be classified into three known karyopherin-alpha subfamilies. We detected mRNA of the four karyopherins in normal 3rd day of 5th instar larvae, and in larvae injected with Grampositive bacteria, Gram-negative bacteria, viruses, and fungi using real-
\end{abstract}


time fluorescence quantitative reverse transcriptase-polymerase chain reaction, and found that the four karyopherins were widely distributed, but their expression levels were related to tissues type, the microbe injected, and the time point.

Key words: Bombyx mori; Karyopherin; Gene clone; Protein expression; Real-time fluorescence quantitative RT-PCR; Immune reaction

\section{INTRODUCTION}

The nuclear envelope (NE) of eukaryotic cells has evolved in order to separate the cytoplasm and the nucleoplasm into two relatively independent regions. Genetic materials are restricted in the nucleus, so protein translation takes place in the cytoplasm. The proteins needed for DNA replication, mRNA transcription, gene regulation, nuclear skeleton repair, or for integrating into the inner nuclear membrane must be transported from the cytoplasm to the nucleoplasm through nuclear-pore complexes (NPCs), which penetrate the NE and connect the cytoplasm with the nucleoplasm. Proteins less than about $40 \mathrm{kDa}$ in weight can go through NPCs directly, but most large proteins need carriers to take them into the nucleus (Pante and Kann, 2002; Terry et al., 2007). Karyopherins, also called importins (IMPs), are important carriers in eukaryotes. There are two types of karyopherins: karyopherin-beta (KPNB), also named importin-beta (IMP $\beta$ ), which can carry cargos through NPCs and interact with FG nucleoporins or Ran-ATP to release their cargoes; and karyopherin-alpha (KPNA), also named importin-alpha (IMPa), is a cargo receptor. KPNA recognizes classic nuclear location sequences (cNLSs) on cargoes and KPNB recognizes importin-beta-binding (IBB) domains on KPNA. Cargoes, KPNA, and KPNB form a ternary complex. The mechanism behind the ability of the complex to go through NPCs is unclear, but probably relates to an interaction between KPNB and nucleoporins (Kapinos et al., 2014). In the nucleus, Ran-ATP binds to KPNB, and the ternary complex disassembles and the cargo is released.

Yeast has one type of KNPA, Drosophila has four, the mouse has six, and other mammals have seven. Based on their molecular characteristics and phylogenetics, KNPAs are divided into three subfamilies, and the cargoes they carry differ (Huenniger et al., 2010; Sachan et al., 2013; Zhou et al., 2013). Yeast, Drosophila, mice, and other mammals all have at least one KPNB. KPNAs and KPNBs play important roles in animal physiology, such as development, immunity, and stress responses (Gorjanacz et al., 2006; Ratan et al., 2008; Wirthmueller et al., 2013; Young et al., 2013; Angus et al., 2014; Schaller et al., 2014). Viruses also use karyopherins to carry their structures, or non-structural proteins, in and out of the nuclei of host cells in order to replicate themselves, e.g., the influenza virus nucleoprotein (Wang et al., 1997; Sasaki et al., 2013). The VP24 proteins coded by the Ebola virus can bind karyopherins to inhibit the immunological responses of an infected cell (Mateo et al., 2010).

The domestic silkworm (Bombyx mori) is one of the most important economic insects in China, Japan, and Southeast Asia, and has been used as a model Lepidopteran (Xia et al., 2004, 2009). We BLAST-searched the domestic silkworm genome database (SilkDB; http://silkworm. genomics.org.cn/) and found three predicted KPNAs (BGIBMGA003758-TA, BGIBMGA011351TA, and BGIBMGA012556-TA) and one predicted KPNB (BGIBMGA002972-TA). We designed primers to clone the four genes, expressed them in Escherichia coli cells, and compared their molecular characteristics, tissue distributions, and expression patterns during immune reactions in the silkworm strain p50. 


\section{MATERIAL AND METHODS}

\section{Experimental animals and treatments}

The domestic silkworm used for the experiments was the p50 strain, which was maintained in the Key Laboratory of Sericultural Biotechnology, Anhui Agriculture University, China. The 5th 3-day-old larvae were divided into five groups, and each group contained at least 16 individuals. The larvae in Group 1 were injected with $4 \mu \mathrm{L}$ phosphate buffer solution (PBS; $\mathrm{pH}, 7.4)$. The larvae in Groups 2 to 5 were injected with $4 \times 10^{6}$ inactivated E. coli, $4 \times 10^{6}$ Beauveria bassiana, $4 \times 10^{6}$ nuclear polyhedrosis virus (NPV), and $2 \mu \mathrm{g}$ Micrococcus luteus, respectively. At the time points of $1,4,8$, and $12 \mathrm{~h}$ after injection, the larvae of Groups 1 to 5 were dissected in order to collect blood and the fat body. Untreated 5th 3-day-old larvae were also dissected, not only to collect blood and the fat body but also the testes, ovaries, Malpighian tubules, midguts, silk glands, and integuments. At each time point, at least four larvae were dissected and the same tissues were mixed together. The experiment was repeated three times.

\section{RNA extraction and cDNA preparation}

Total RNA from the above tissues was extracted using the TRIzol method (Takara, Dalian, China). RNA quantity and quality were checked by detecting the values of OD260 and OD280, and also by RNA electrophoresis. The cDNA used for the cloned genes was produced using a TransScript ${ }^{\circledR}$ First-Strand cDNA Synthesis SuperMix (Transgen, Beijing, China), and the cDNA used for the real-time fluorescence quantitative reverse transcriptase-polymerase chain reaction (RT-PCR) was produced using a PrimeScript ${ }^{\mathrm{TM}}$ RT Master Mix (Takara, Dalian, China).

\section{Cloning and real-time fluorescence quantitative RT-PCR primer design}

Primers were designed using Primer Premier 5.0 software, and the PCRs were verified by DNA sequencing. Real-time fluorescence quantitative RT-PCR primers of $B$. mori 18s rRNA have been reported previously (Zhang et al., 2012). The primers and their annealing temperatures are listed in Table 1.

\section{Plasmid construction and recombinant prokaryotic protein expression}

Open reading frames (ORFs) of the Bm-KPNAs and Bm-KPNB1 were cloned in a pGEM ${ }^{\circledR}-\mathrm{T}$ Easy Vector (Transgen) and amplified in Trans-T1 cells (Transgen). Plasmids were extracted using an AxyPrep ${ }^{\text {TM }}$ Plasmid Miniprep Kit (Axygen, Suzhou, China). Bm-KPNA1, Bm-KPNA3, and BmKPNB1 were double-digested by BamHI/ Xhol (Takara), and KPNA2 was digested by BamHI/ Sall (Takara) at $37^{\circ} \mathrm{C}$ for $4 \mathrm{~h}$. The prokaryote expression plasmid pET-28a+ was also doubledigested by the above enzyme pairs, under similar conditions. Target fragments were collected by agarose electrophoresis and purified using an AxyPrep ${ }^{\mathrm{TM}}$ DNA Gel Extraction Kit (Axygen). The targeted gene fragments and pET-28a+ were linked by T4 ligase (Takara) at $25^{\circ} \mathrm{C}$ for $1 \mathrm{~h}$. The recombinant plasmids were verified by DNA sequencing and transferred into Transetta (DE3) cells (Transgen). Recombinant protein expression was induced by different concentrations of isopropyl $\beta$-D-1-thiogalactopyranoside (IPTG) (Sangon, Shanghai, China) at $30^{\circ} \mathrm{C}$ for $4 \mathrm{~h}$. 


\begin{tabular}{|c|c|c|c|}
\hline Gene name & Primer sequence (5' to $\left.3^{\prime}\right)$ & Annealing temperature & PCR product size (bp) \\
\hline \multicolumn{4}{|c|}{ Open reading frame primers } \\
\hline \multirow[t]{2}{*}{ Bm-KPNA1 } & F: CGCGGATCCATGTCTGGAGGTCAC & $\begin{array}{l}58^{\circ}-0.2^{\circ} \mathrm{C} / \text { cycle } \\
35 \text { cycles }\end{array}$ & 1581 \\
\hline & R: CCGCTCGAGGTTAAAACTGAAAGC & & \\
\hline \multirow[t]{2}{*}{ Bm-KPNA2 } & F: CGCGGATCCATGGCTGAACGAACCTCG & $\begin{array}{l}60^{\circ}-0.2^{\circ} \mathrm{C} / \text { cycle } \\
40 \text { cycles }\end{array}$ & 1533 \\
\hline & R: GACGTCGACCTAGAAGTGTATGGCTGC & & \\
\hline \multirow[t]{2}{*}{ BM-KPNA3 } & F: CGC & $\begin{array}{l}60^{\circ}-0.2^{\circ} \mathrm{C} / \text { cycle } \\
40 \text { cycles }\end{array}$ & 1569 \\
\hline & R: CCGCTCGAGCTAGAAGCGGA & & \\
\hline \multirow[t]{2}{*}{ Bm-KPNB1 } & F: CGCGGATCCATGCACGCAGAAAC & $\begin{array}{c}58^{\circ} \mathrm{C} \\
30 \text { cycles }\end{array}$ & 2679 \\
\hline & R: CCGCTCGAGTTACCAGCTGGCAAGAGG & & \\
\hline \multicolumn{4}{|c|}{$\begin{array}{l}\text { Real-time fluorescence } \\
\text { quantitative RT-PCR primers }\end{array}$} \\
\hline Bm-KPNA1 & $\begin{array}{l}\text { F: GTTCTCCCACCCTTGCTT } \\
\text { R: TTTCTCGTTCGGACCATCT }\end{array}$ & $60^{\circ} \mathrm{C}$ & 231 \\
\hline BM-KPNA2 & $\begin{array}{l}\text { F: CAGGCTGCGGCGAAATAC } \\
\text { R: GAACTGATACTGCTCGTGGTC }\end{array}$ & $60^{\circ} \mathrm{C}$ & 201 \\
\hline BM-KPNA3 & $\begin{array}{l}\text { F: TGATTCCGTTGTTGTCGC } \\
\text { R: GTTTATTACCTGCCGTGATGT }\end{array}$ & $60^{\circ} \mathrm{C}$ & 210 \\
\hline Bm-KPNB1 & $\begin{array}{l}\text { F: TGCTTTGAGTATTGGTAGCGATTT } \\
\text { R: GTCGTACCTGAGAACCTACACCT }\end{array}$ & $60^{\circ} \mathrm{C}$ & 194 \\
\hline $\mathrm{Bm}-18 \mathrm{~S}$ & $\begin{array}{l}\text { F: CGATCCGCCGACGTTACTACA } \\
\text { R: GTCCGGGCCTGGTGAGATTT }\end{array}$ & $60^{\circ} \mathrm{C}$ & 180 \\
\hline
\end{tabular}

Underlined text refers to enzyme digestion sites.

\section{Protein electrophoresis and western blot analysis}

The cells containing recombinant proteins were centrifuged at 12,000 rpm for $1 \mathrm{~min}$. After adding $24 \mu \mathrm{L}$ PBS and $6 \mu \mathrm{L} 5 \mathrm{X}$ protein loading buffer, the precipitates were placed in boiling water for $10 \mathrm{~min}$ before being stored in ice. Subsequently, $10 \mu \mathrm{L}$ of the protein solution was used for $12 \%$ polyacrylamide gel electrophoresis (PAGE) and dyed with Coomassie brilliant blue R250 (Sangon), or used for western blot analysis.

Western blot analysis was initiated by transferring the proteins from the PAGE gels to polyvinylidene difluoride films (Millipore, USA). The films were washed with PBS with Tween ${ }^{\circledR}$ 20 (PBST) three times, each time for $10 \mathrm{~min}$. A non-specific antibody recognized sites on the films blocked with $5 \%$ fat-free milk powder, and the films were then incubated with mouse anti-His monoclonal antibody (Transgen) for $1 \mathrm{~h}$ at room temperature. The films were washed with PBST another three times, and then incubated with HRP-labeled goat anti-mouse polyclonal antibody (Transgen). After again being washed three times with PBST, the films were colored using a DAB kit (Sangon).

\section{Bioinformatics analysis}

The proteins' molecular weights and isoelectric points were analyzed using the ExPASY Compute $\mathrm{pl} / \mathrm{Mw}$ tool (http://web.expasy.org/compute_pi/). Domains and repeats were analyzed using Pfam (http://pfam.xfam.org/) (Punta et al., 2012; Finn et al., 2014). KPNA and KPNB protein sequences from different organisms were collected from online protein databases such as GenBank (http://www.ncbi.nlm.nih.gov/genbank/), MGI (http://www.informatics.jax.org/), FlyBase 
(http://flybase.org/), and BeeBase (http://hymenopteragenome.org/beebase/) for phylogenetic analysis. Multiple sequence alignments were conducted using ClustalX 2.1, and phylogenic trees were constructed based on amino acid (AA) sequences by MEGA 6.06, using the neighbor-joining method with a bootstrap test of 1000 replications (Tamura et al., 2011).

\section{Statistical analysis}

Statistical analysis was performed using PASW Statistics 18.0. Means were compared by one-way analyses of variance (ANOVA), and post-hoc multiple comparisons were conducted using the least significant difference (LSD) method. The significance level was set at 0.05 , and each experiment was repeated at least three times.

\section{RESULTS}

\section{Clones and sequence analysis}

The cloned ORF sequences of Bm-KPNA1, Bm-KPNA2, Bm-KPNA3, and Bm-KPNB1 were 1563, 1515, 1551, and 2661 bp long, respectively, and coded 520, 504, 516, and 886 AAs, respectively. The Bm-KPNA ORF sequences were identical to BGIBMGA011351-TA, BGIBMGA011351-TA, and BGIBMGA012556-TA. The Bm-KPNB1 ORF was $96.06 \%$ similar to BGIBMGA002972-TA, and its AA sequence similarity was 95.49\%. BGIBMGA002972-TA was short of two intron-like regions when aligned with BM-KPNB1. One was $40 \mathrm{bp}$ long, and followed a "T" inserted after the 25th nucleotide (Figure 1A); the other was 63 bp long (Figure 1B). The nucleotides of the two regions were integer multiples of three, so there were only 19 and 21 AAs difference (Figure 1C, D).

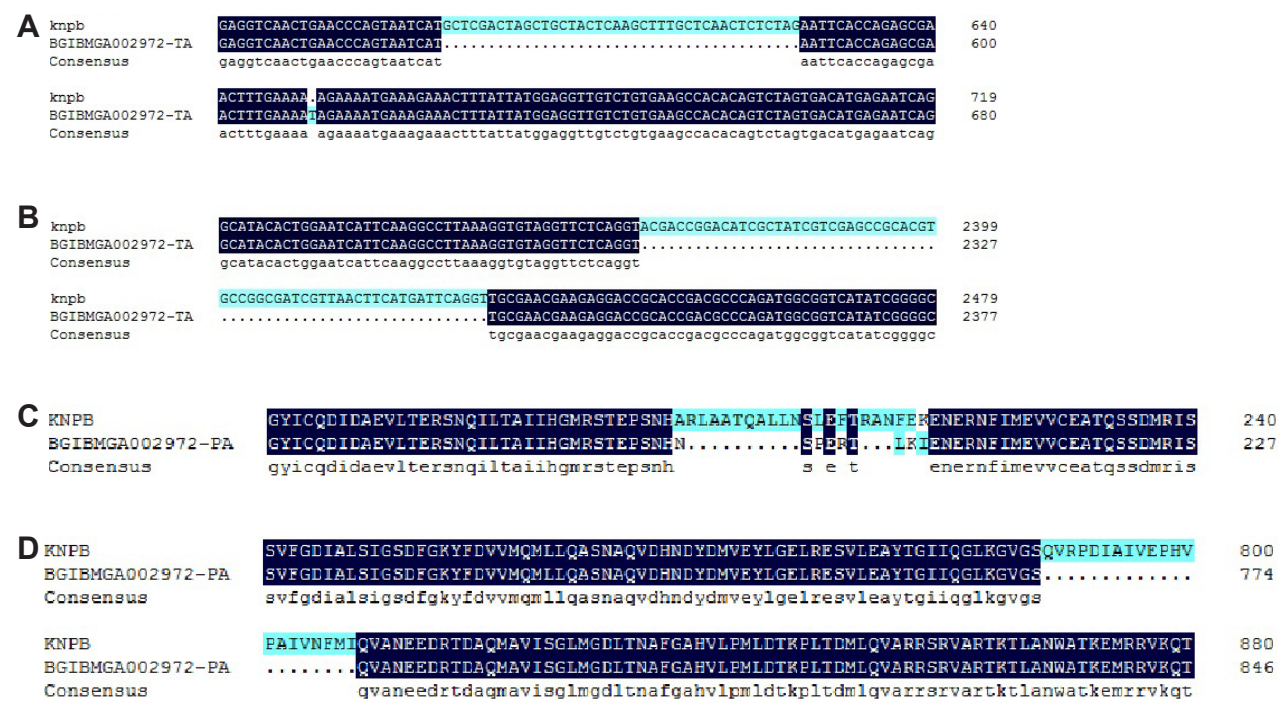

Figure 1. Nucleotide and amino acid sequences aligned between Bm-KPNB1 and BGIBMGA002972 on SilkDB; A and $\mathbf{B}$ show the nucleotide differences, $\mathbf{C}$ and $\mathbf{D}$ show the amino acid differences. 
The aligned ORFs and protein sequences of the Bm-KPNAs revealed that the similarities between Bm-KPNA1 and BM-KPNA3 (52.16 and 45.71\%, respectively) and BM-KPNA2 (49.21 and $40.65 \%$, respectively) were higher than those between Bm-KPNA2 and Bm-KPNA3 (47.76 and $38.34 \%$, respectively). These results support the hypothesis that KPNA2 and KPNA3 developed from KPNA1 (Mason et al., 2009). Bm-KPNB1 had the lowest similarity with the KPNAs (Table 2).

Table 2. Similarity (\%) of open reading frames and protein sequences between Bm-KPNAs and Bm-KPNB1.

\begin{tabular}{lcccc}
\hline & Bm-KPNA1 & Bm-KPNA2 & Bm-KPNA3 & Bm-KPNB1 \\
\hline Bm-KPNA1 & $100 / 100$ & - & - & - \\
Bm-KPNA2 & $49.21 / 40.65$ & $100 / 100$ & - & - \\
Bm-KPNA3 & $52.16 / 45.71$ & $47.76 / 38.34$ & $100 / 100$ & - \\
Bm-KPNB1 & $24.46 / 8.24$ & $23.32 / 8.13$ & $23.90 / 10.15$ & $100 / 100$ \\
\hline
\end{tabular}

Fifty-two KPNAs, belonging to 20 animal species from nine orders of three phyla, were collected to construct the phylogenic tree. The insects had three KPNAs, except for Drosophila melanogaster and Pararge aegeria, which had four and two, respectively. These KPNAs were classified into three clades that represented different alpha subfamilies, and each Bm-KPNA was classified into one of the subfamilies. Therefore, we named Bm-KPNAs according to the subfamily they belonged to (Figure 2). BM-KPNA1 and BM-KPNA2 were most similar to $P$. aegeria (81.6 and $68.2 \%$, respectively), while KPNA3 was most similar to $N$. vitripennis (77.5\%). Twenty-one KPNBs, from seven orders of three phyla that were also aligned, were similar. BM-KPNB1 was most similar to Danaus plexippus (86.3\%) (Figure 3).

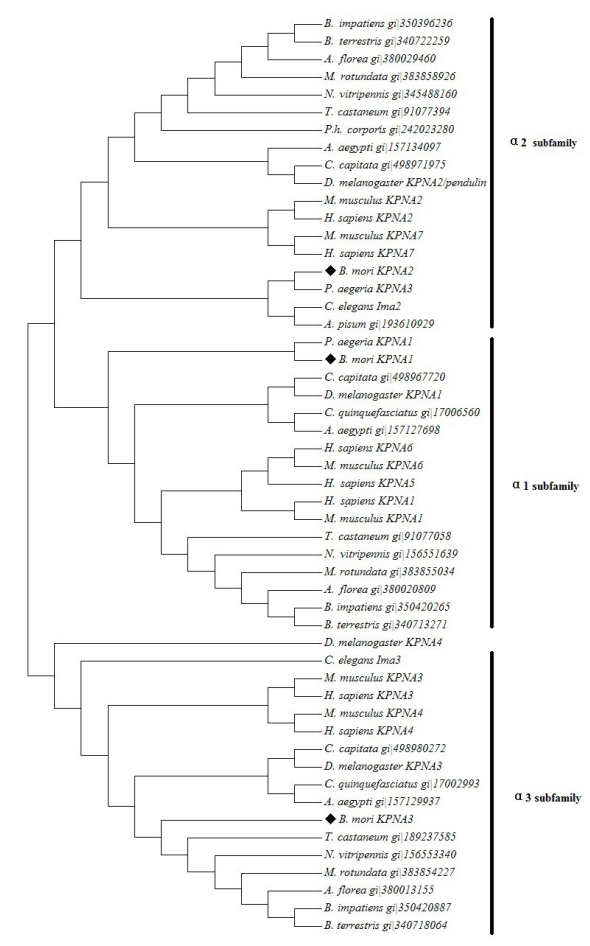

Figure 2. Phylogenic tree of KPNAs constructed using the neighbor-joining algorithm method. 


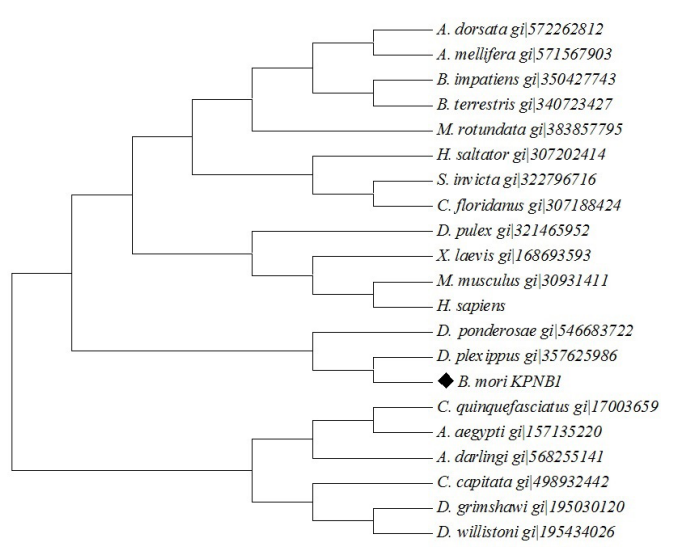

Figure 3. Phylogenic tree of KPNBs constructed using the neighbor-joining algorithm method.

KPNA1 had 201 identical AAs (Figure 4), KPNA2 had 68 (Figure 5), KPNA3 had 95 (Figure 6 ), and KPNB had 316 (Figure 7), which accounted for 38.65, 13.49, 18.41, and 35.66\%, respectively. These results suggest that KPNA1 and KPNB were under greater evolutionary pressure than were KPNA2 or KPNA3. These AAs must play important roles for proteins to perform their normal functions.
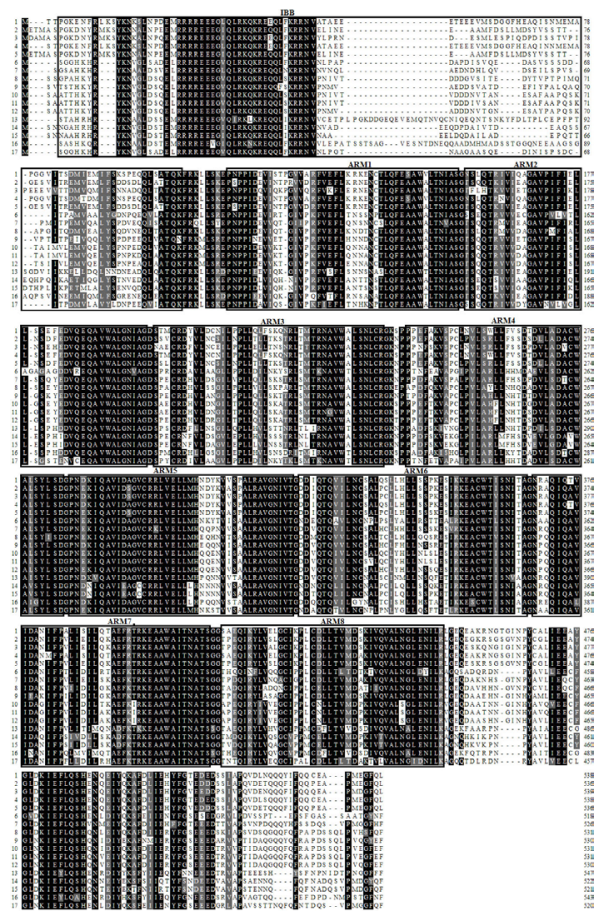

Figure 4. Multiple alignments between 17 KPNA1. 1, Homo sapiens KPNA1; 2, H. sapiens KPNA6; 3, H. sapiens KPNA5; 4, Mus musculus KPNA1; 5, M. musculus KPNA6; 6, Pararge aegeria KPNA1; 7, Tribolium castaneum gi|91077058; 8, Nasonia vitripennis gi|156551639; 9, Megachile rotundata gi|383855034; 10, Bombus impatiens gi|350420265; 11, Bombus terrestris gi|340713271; 12, Apis florea gi|380020809; 13, Ceratitis capitata gi|498967720; 14, Culex quinquefasciatus gi|170065607; 15, Aedes aegypti gi|157127698; 16, Drosophila melanogaster KPNA1; 17, Bombyx mori KPNA1. 

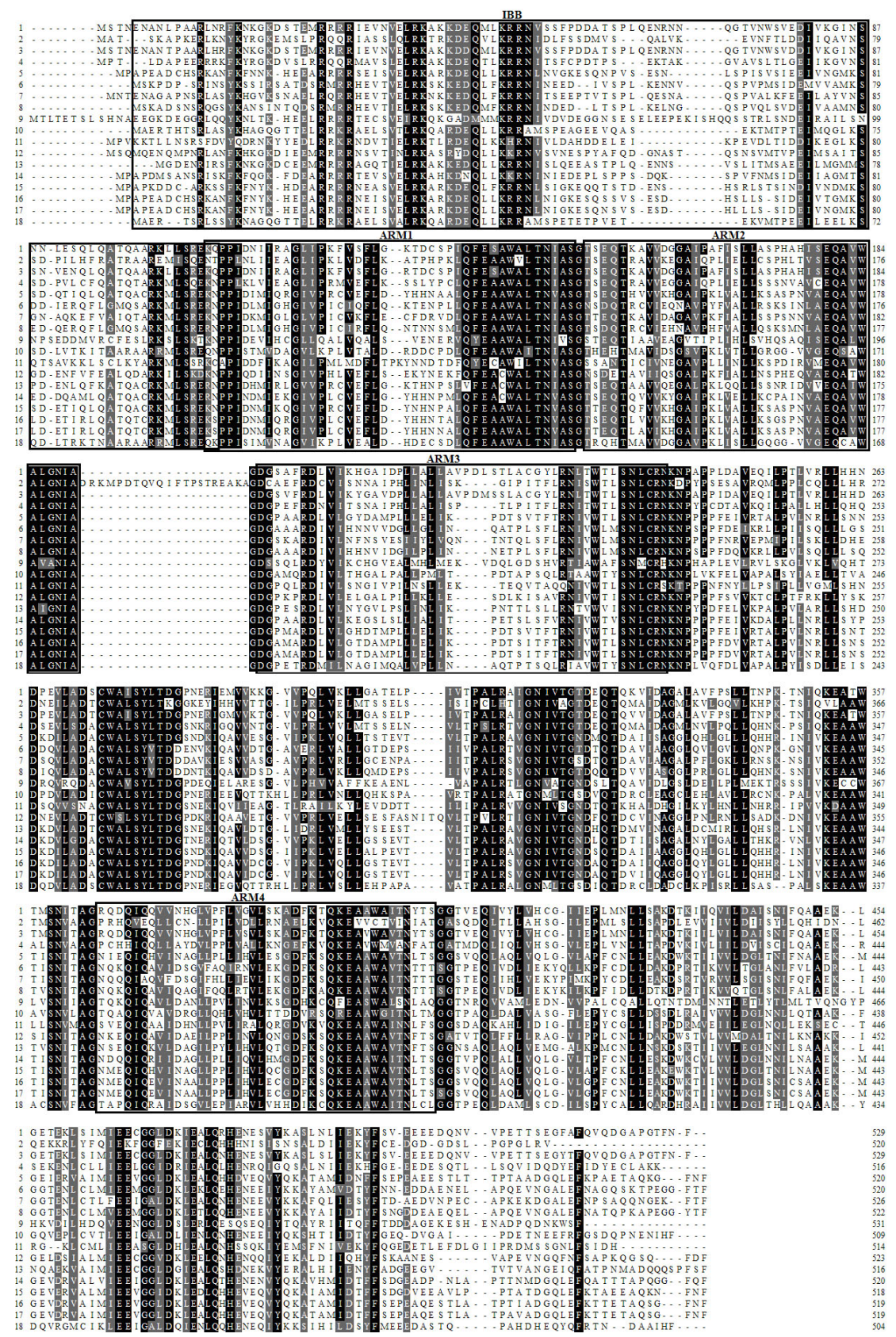

529
520
529
516
520
520
526
522
531
509
514
523
516
520
518
519
519
504

Figure 5. Multiple alignments between 18 KPNA2. 1, Mus musculus Kpna2; 2, M. musculus Kpna7; 3, Homo sapiens KPNA2; 4, H. sapiens KPNA7; 5, Apis florea gi|380029460; 6, Ceratitis capitata gi|498971975; 7, Aedes aegypti gi|157134097; 8, Drosophila melanogaster KPNA2/pendulin; 9, Caenorhabditis elegans Ima2; 10, Pararge aegeria KPNA3; 11, Acyrthosiphon pisum gi|193610929; 12, Pediculus humanus corporis gi|242023280; 13, Tribolium castaneum gi|91077394; 14, Nasonia vitripennis gi|345488160|; 15, Megachile rotundata gi|383858926; 16, Bombus impatiens gi|350396236; 17, Bombus terrestris gi|340722259; 18, Bombyx mori KPNA2. 

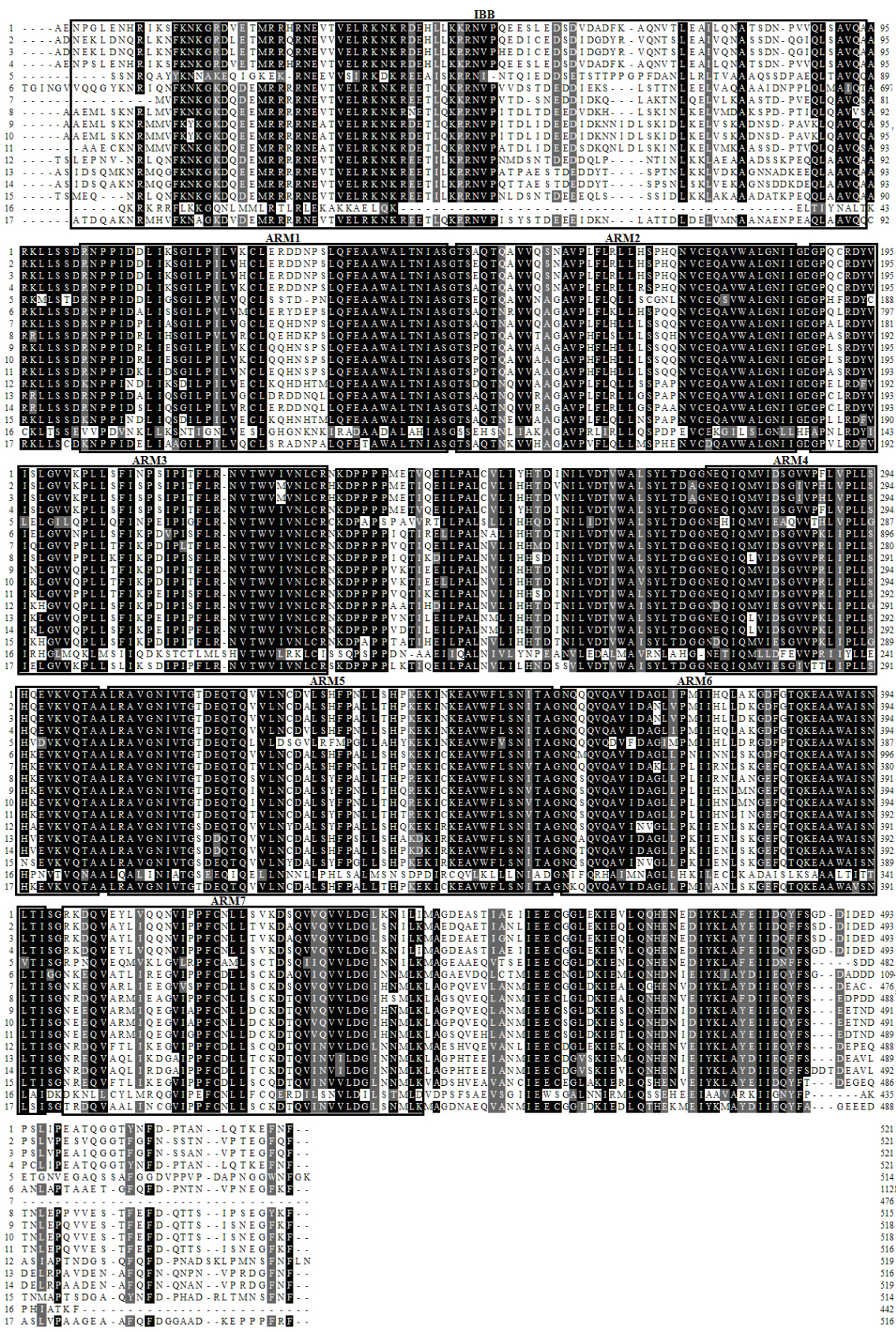

521
521
521
521
514
1121
476
511
518
518
518
516
519
516
519
514
442
516

Figure 6. Multiple alignments between 17 KPNA3. 1, Mus musculus KPNA3; 2, M. musculus KPNA4; 3, Homo sapiens KPNA4; 4, H. sapiens KPNA3; 5, Caenorhabditis elegans Ima-3; 6, Tribolium castaneum gi|189237585; 7, Nasonia vitripennis gi|156553340; 8, Megachile rotundata gi|383854227; 9, Bombus impatiens gi|350420887; 10, Bombus terrestris gi|340718064; 11, Apis florea gi|380013155; 12, Ceratitis capitata gi|498980272; 13, Culex quinquefasciatus gi|170029939; 14, Aedes aegypti gi|157129937; 15, Drosophila melanogaster KPNA3; 16, D. melanogaster KPNA4; 17, Bombyx mori KPNA3. 

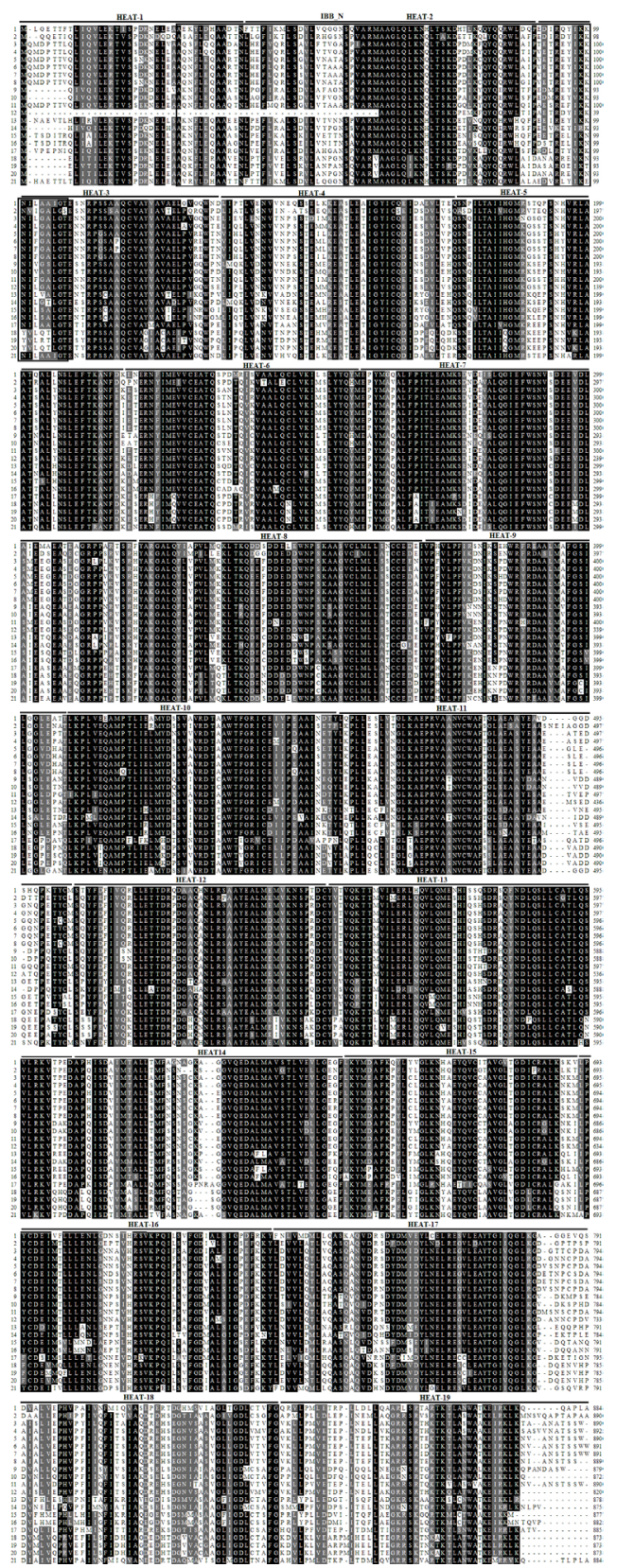

Figure 7. Multiple alignments between 21 KPNB. 1, Danaus plexippus gi|357625986; 2, Dendroctonus ponderosae gi|546683722; 3, Harpegnathos saltator gi|307202414; 4, Solenopsis invicta gi|322796716; 5, Bombus impatiens gi|350427743; 6, Apis dorsata gi|572262812; 7, Apis mellifera gi|571567903; 8, Bombus terrestris gi|340723427; 9 , Culex quinquefasciatus gi|170036594; 10, Aedes aegypti gi|157135220; 11, Megachile rotundata gi|383857795; 12, Cryptoramorphus floridanus gi|307188424; 13, Ceratitis capitata gi|498932442; 14, Anopheles darlingi gi|568255141; 15, Drosophila grimshawi gi|195030120; 16, Drosophila willistoni gi|195434026; 17, Daphnia pulex gi|321465952; 18, Mus musculus gi|30931411; 19, Xenopus laevis gi|168693593; 20, Homo sapiens KPNB; 21, Bombyx mori KPNB. 
By analyzing the protein sequences using Pfam, we found that the Bm-KPNAs all had one IBB domain, and Bm-KPNA1, 2, and 3 had 8, 4, and 7 armadillo/beta-catenin-like repeats (ARMs), respectively (Figures 4, 5, and 6). Bm-KPNB1 had 19 HEAT repeats; Heat_2, part of HEAT_1 and HEAT_3, constructed an importin-beta-N-terminal domain (IBB_N), and HEAT_7 to HEAT_19 constructed an IBB-binding domain (Figure 7).

\section{Prokaryotic protein expression}

Cells with recombinant BM-KPNAs or BM-KPNB1 plasmids induced by IPTG at $30^{\circ} \mathrm{C}$ could correctly express recombinant proteins. The molecular weight of recombinant Bm-KPNAs was about $60 \mathrm{kDa}$, and that of BM-KPNB1 was about $100 \mathrm{kDa}$, which is in accord with the molecular weights predicted by ExPASY (Figure 8).

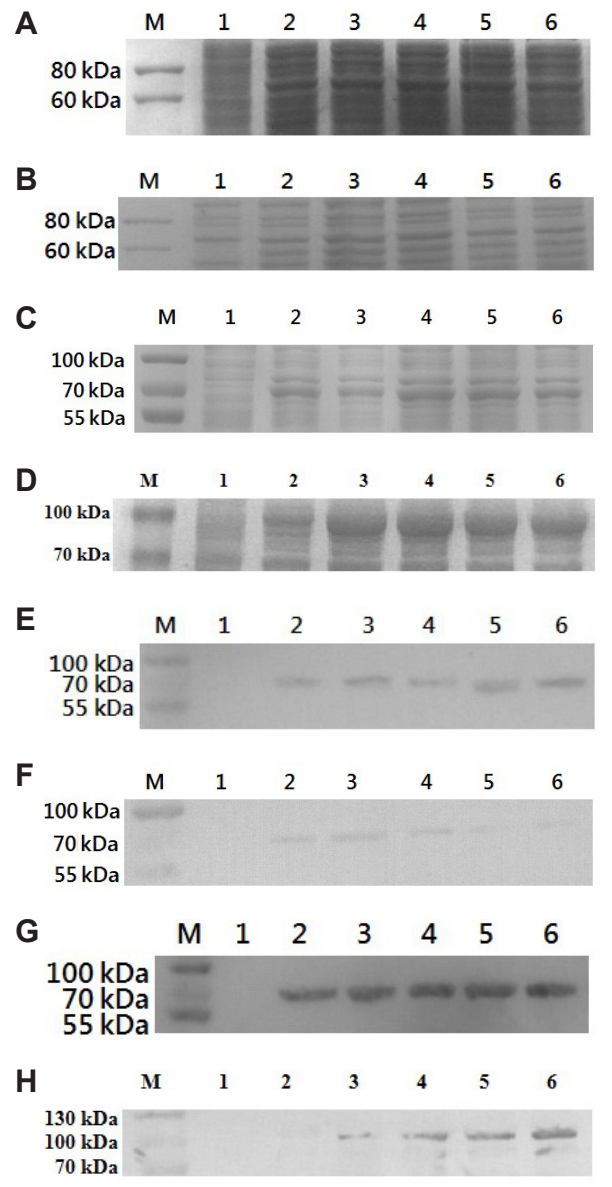

Figure 8. Recombinant protein expression of BM-KPNAs and BM-KPNB1. A to D represent BM-KPNA1, 2, 3, and Bm-KPNB1 recombination proteins dyed with Coomassie brilliant blue R-250; $\mathbf{E}$ to $\mathbf{H}$ represent western blot results of BM-KPNA1, 2, 3, and Bm-KPNB1 recombinant proteins. Lanes $M, 1,2,3,4,5$, and $6=$ protein markers, cells with blank expression vectors, or cells with gene targeting induced by $0,0.3,0.6,0.9$, or $1.2 \mathrm{mM}$ isopropyl $\beta$-D-1thiogalactopyranoside, respectively. 


\section{Tissue expression analysis}

We checked Bm-KPNA and Bm-KPNB1 mRNA levels in the fat body, silk glands, Malpighian tubules, hemocytes, midguts, testes, ovaries, and integuments of 5th 3-day-old larvae, and found that the four kinds of protein were expressed in all eight tissue types, but the expression levels varied (Figure 9). The highest expression of all the proteins occurred in the testes. BM-KPNA2 was expressed approximately twice as much in the testes as were the other proteins, and its expression was 846.2-fold higher than that in the silk gland.

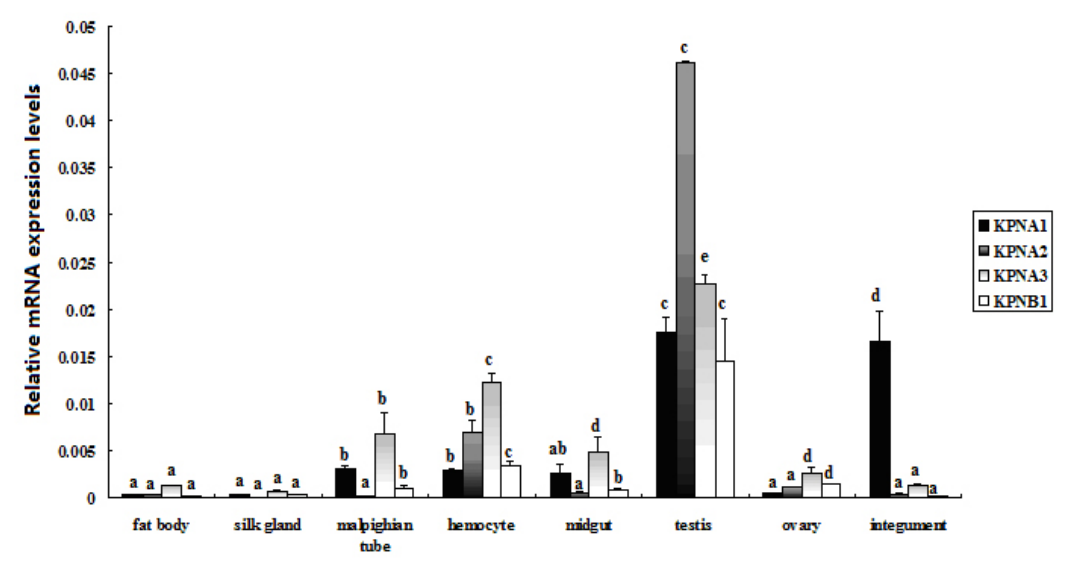

Figure 9. Relative mRNA expression levels of Bm-KPNAs and Bm-KPNB1. The 18s rRNA levels were defined as 1. Different letters represent significant differences $(P<0.05)$.

\section{Relative mRNA levels in response to microbial injections}

The relative mRNA levels of the Bm-KPNAs and Bm-KPNB1 in response to the microbes differed. In the fat body, Bm-KPNA mRNA levels were generally low at 1 and $8 \mathrm{~h}$, but increased at 4 and $12 \mathrm{~h}$. Bm-KPNA1 was most sensitive to E. coli, B. bassiana, and NPV, Bm-KPNA3 to M. luteus, E. coli, and B. bassiana, and Bm-KPNA2 was not as sensitive as the other two Bm-KPNAs to the four microbes. Bm-KPNB1 $\mathrm{mRNA}$ levels in response to $E$.coli and $M$. luteus exhibited no significant variation at 1,4 or $8 \mathrm{~h}$, but at $12 \mathrm{~h}$ they increased by 2.97 -fold and 4.93 -fold, respectively. When responding to $B$. bassiana and NPV, Bm-KPNB1 mRNA levels slightly fluctuated. In hemocytes, Bm-KPNA and Bm-KPNB1 mRNA levels were first downregulated and then upregulated, before decreasing again at $4 \mathrm{~h}$ to basal levels. They were most sensitive to $B$. bassiana, and were upregulated to $15.54,19.32$, 8.09, and 3.93-fold compared with control groups, respectively, at $8 \mathrm{~h}$ (Figure 10).

\section{DISCUSSION}

SilkDB predicted three Bm-KPNA ORFs and one Bm-KPNB1 ORF. We designed primers to amplify the four protein ORFs, and the Bm-KPNA sequencing results matched the sequences on SilkDB. The Bm-KPNB1 ORF was shorter (102 bp) than the sequence on SilkDB. Bm-KPNB1 probably has variable expression modes that code for a long and a short peptide. We designed PCR primers to amplify the two different regions, and tested them on $15 \%$ polyacrylamide gel; after testing 30 silkworms, we did not find the short Bm-KPNB1 (Figure 11). 
A

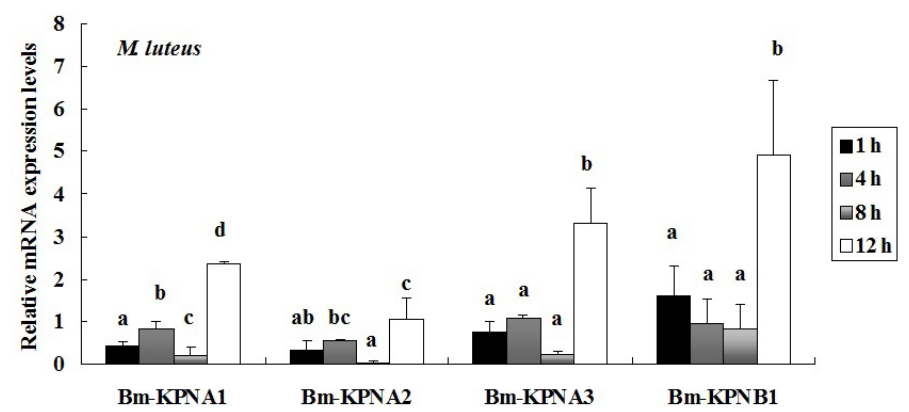

B

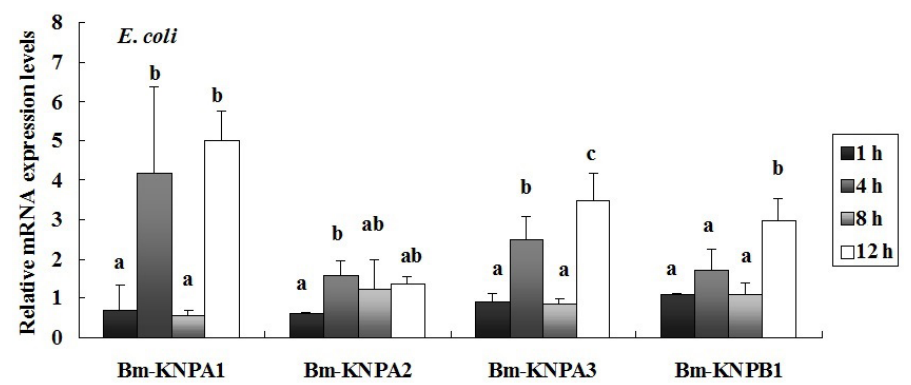

C

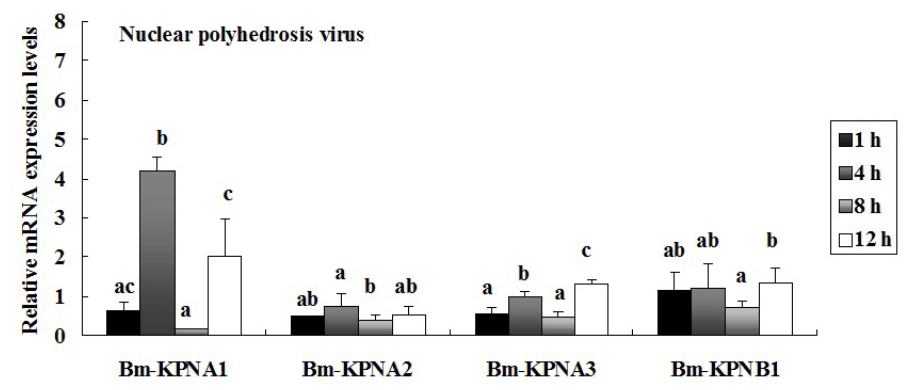

D

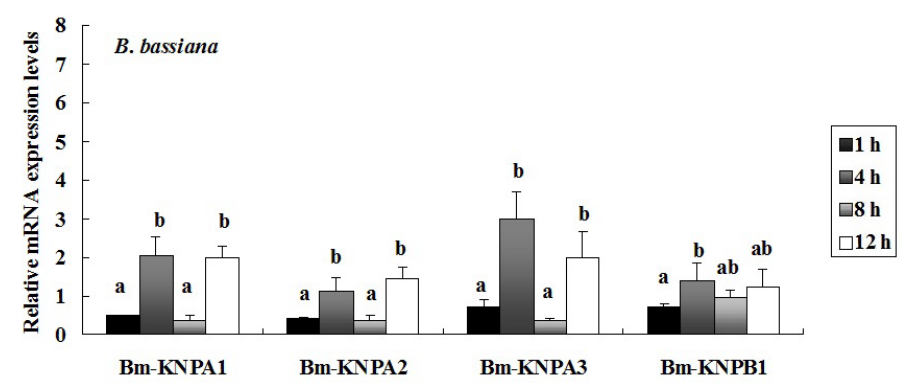

Figure 10. Relative mRNA expression levels of Bm-KPNAs and Bm-KPNB1 in response to four kinds of microbe. $\mathbf{A}$ to $\mathbf{D}$ represent responses in the fat body, $\mathbf{E}$ to $\mathbf{H}$ represent responses in the hemocyte. At each time point, the corresponding mRNA expression levels of larvae injected with PBS were defined as 1 . Different letters represent significant differences $(P<0.05)$.

Continued on next page 
Figure 10. Continued.
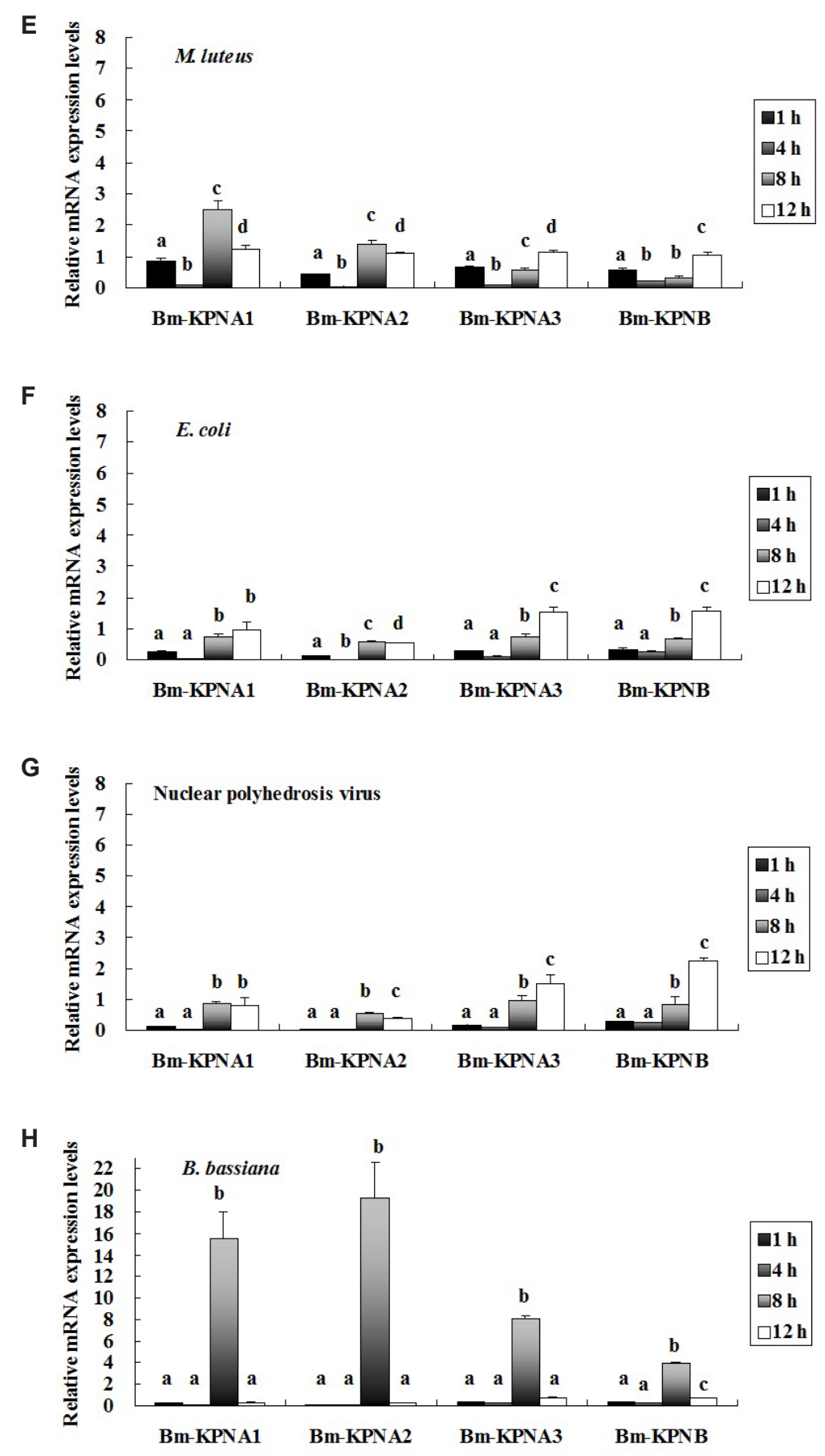

Genetics and Molecular Research 14 (4): 13049-13065 (2015) 


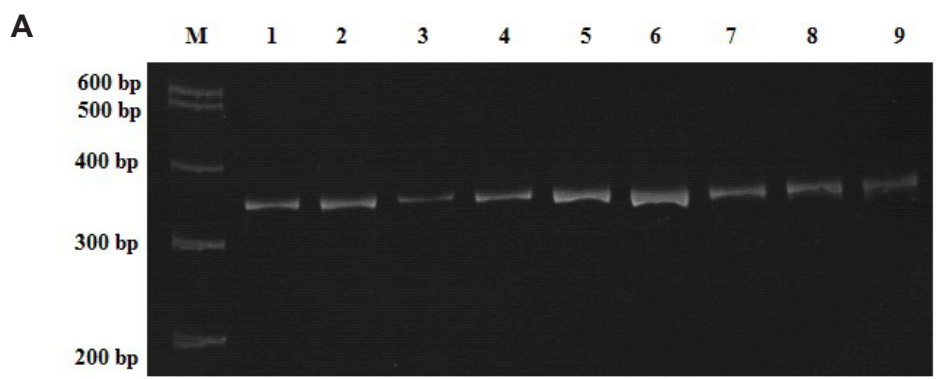

B

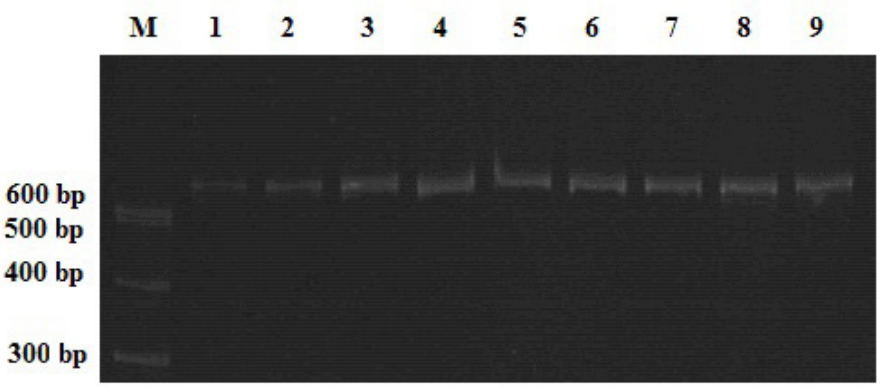

Figure 11. DNA native polyacrylamide gel electrophoresis (Native-PAGE) of Bm-KPNB1. M represents a DNA marker. 1 to 9 represent cDNA templates from different larvae; each cDNA template was a cDNA pool from the sex glands, Malpighian tubules, midguts, silk glands, fat bodies, hemocytes, and integuments from 5th 3-day-old larvae. A. amplified from 470 to $893 \mathrm{bp}$; B. amplified from 1879 to $2550 \mathrm{bp}$ of the Bm-KPNB1 open reading frame. A. forward primer 5'-TGGAGGCTATTGGCTACATT-3' and reverse primer 5'-TCTATTTCCTCATCGCTCACA-3'; B. forward primer 5'-GCAGGCAGTGTTCAGGAGGA-3' and reverse primer 5'-GTCGGTGAGCGGCTTGGTGT-3'. The annealing temperature of the two pairs of primers was $60^{\circ} \mathrm{C}$.

Animal KPNAs are divided into three conserved subfamilies, named $\alpha 1$, $\alpha 2$, and $\alpha 3$ (Köhler et al., 1997; Mason et al., 2009). The KPNAs of the domestic silkworm are also classed into three conserved subfamilies, and we named them karyopherin a1 (BM-KPNA1), karyopherin a2 (BM-KPNA2), and karyopherin a3 (BM-KPNA3). Humans contain seven KPNAs, and KPNA1, 5 , and 6 are in the a1 subfamily, KPNA2 and 7 are in the a2 subfamily, and KPNA3 and 4 are in the a3 subfamily (Köhler et al., 1997; Mason et al., 2009; Kelley et al., 2010; Yang et al., 2010). The phylogenic tree we constructed confirmed this; however, P. aegeria KPNA3 was a special case, for it is named KPNA3 but is in the a2 clade. To date, only three KPNAs have been found in insects, but Drosophila has an additional Dmel-KPNA4 (FlyBase, FBpp0076777). We found that Dmel-KPNA4 has not an obvious IBB domain, but other Dmel-KPNAs all have one IBB domain.

KPNAs and KPNBs in different organisms have conserved domains, which can identify them from other proteins. KPNAs have IBB and ARM repeats, and KPNBs have IBB_N, IBBbinding domain, and HEAT repeats. ARMs and HEATs are approximately 50 AA-long tandem repeated sequence motifs that probably have a common phylogenetic origin (Andrade et al., 2001). Several ARMs and HEATs construct protein-protein interaction domains, but no common features among the target proteins could be found. The domains constructed by ARMs in KPNAs recognize NLSs, and those constructed by HEATs in KPNBs recognize the IBB of KPNA and other proteins to be transported into the nucleus (Cingolani et al., 1999; Lee et al., 2003; Mynott et al., 2011). Many nuclear pore proteins contain FG sequence repeats that can bind to HEAT repeats within KNPBs, 
which is important for KNPB -mediated transport (Vetter et al., 1999; Bayliss et al., 2002; Isgro and Schulten, 2007; Lange et al., 2007). The IBB_N of KPNBs overlaps with HEATS, and interacts with Ran-GTP and Ran-GDP, which is important for cargo-KPNA-KPNB complex disassembly and KPNB relocation to the cytoplasm (Vetter et al., 1999). Similar to other KPNAs, Bm-KPNAs all have one IBB and several ARMs. Bm-KPNB1 has one IBB_N and 19 HEATs.

Silkworms lack adaptive immune systems, and possess innate immunity that only responds to common bacterial, fungal, and viral pathogens. Their responses involve cellular mechanisms, such as programmed cell death or autophagy, and the expression of a large set of genes. Our results show that Bm-KPNA and Bm-KPNB1 mRNA expression levels vary according to the pathogen, since $E$. coli is a Gram negative bacteria, M. luteus is a Gram positive bacteria, $B$. bassiana is a fungus, and NPV is a virus.

KNPAs and KNPBs are expressed a great deal in silkworm larvae, indicating that they play important roles in basic physiological processes. The fact that expression levels differed between tissues suggests that they probably play different roles in different tissues (Kimura and Imamoto, 2014). The probable reason that Bm-KPNA2 mRNA expression levels were high in the testes is that this organ is highly active, and KPNA2 interacts with proteins that are associated with mRNA processing, ribonucleoprotein complex biogenesis, chromatin modification, and transcription (Huang et al., 2013; Umegaki-Arao et al., 2013). Since the karyopherin family transports cargoes through the NE, and some cargoes are probably gene expression regulation factors, it is important to ascertain what cargoes they transport in different tissues.

\section{ACKNOWLEDGMENTS}

Research supported by the earmarked Fund for Modern Agro-Industry Technology Research System (CARS-22 SYZ10), the Biology Key Subjects of Anhui Province, the National "863" Plans Project of China (\#2011AA100306), the Sericulture Biotechnology Innovation Team (\#2013xkdt-05), PhD programs in Biochemistry and Molecular Biology (\#xk2013042), and the Natural Science Foundation of the Anhui Higher Education Institutions of China (\#KJ2014A071).

\section{REFERENCES}

Andrade MA, Petosa C, O'Donoghue SI, Muller CW, et al. (2001). Comparison of ARM and HEAT protein repeats. J. Mol. Biol. 309: 1-18.

Angus L, van der Watt PJ and Leaner VD (2014). Inhibition of the nuclear transporter, Kpnbeta1, results in prolonged mitotic arrest and activation of the intrinsic apoptotic pathway in cervical cancer cells. Carcinogenesis 35: 1121-1131.

Bayliss R, Littlewood T, Strawn LA, Wente SR, et al. (2002). GLFG and FxFG nucleoporins bind to overlapping sites on importin-beta. J. Biol. Chem. 277: 50597-50606.

Cingolani G, Petosa C, Weis K and Muller CW (1999). Structure of importin-beta bound to the IBB domain of importin-alpha. Nature 399: 221-229.

Finn RD, Bateman A, Clements J, Coggill P, et al. (2014). Pfam: the protein families database. Nucleic Acids Res. 42: D222-D230.

Gorjanacz M, Torok I, Pomozi I, Garab G, et al. (2006). Domains of Importin-alpha2 required for ring canal assembly during Drosophila oogenesis. J. Struct. Biol. 154: 27-41.

Huang L, Wang HY, Li JD, Wang JH, et al. (2013). KPNA2 promotes cell proliferation and tumorigenicity in epithelial ovarian carcinoma through upregulation of c-Myc and downregulation of FOXO3a. Cell Death Dis. 4: e745.

Huenniger K, Kramer A, Soom M, Chang I, et al. (2010). Notch1 signaling is mediated by importins alpha 3, 4, and 7. Cell Mol. Life Sci. 67: 3187-3196.

Isgro TA and Schulten K (2007). Association of nuclear pore FG-repeat domains to NTF2 import and export complexes. J. Mol. Biol. 366: 330-345. 
Kapinos LE, Schoch RL, Wagner RS, Schleicher KD, et al. (2014). Karyopherin-centric control of nuclear pores based on molecular occupancy and kinetic analysis of multivalent binding with FG nucleoporins. Biophys. J. 106: 1751-1762.

Kelley JB, Talley AM, Spencer A, Gioeli D, et al. (2010). Karyopherin alpha7 (KPNA7), a divergent member of the importin alpha family of nuclear import receptors. BMC Cell Biol. 11: 63.

Kimura M and Imamoto N (2014). Biological significance of the importin-beta family-dependent nucleocytoplasmic transport pathways. Traffic 15: 727-748.

Köhler M, Ansieau S, Prehn S, Leutz A, et al. (1997). Cloning of two novel human importin- $\alpha$ subunits and analysis of the expression pattern of the importin- $\alpha$ protein family. FEBS Lett. 417: 104-108.

Lange A, Mills RE, Lange CJ, Stewart M, et al. (2007). Classical nuclear localization signals: definition, function, and interaction with importin alpha. J. Biol. Chem. 282: 5101-5105.

Lee SJ, Sekimoto T, Yamashita E, Nagoshi E, et al. (2003). The structure of importin-beta bound to SREBP-2: nuclear import of a transcription factor. Science 302: 1571-1575.

Mason DA, Stage DE and Goldfarb DS (2009). Evolution of the metazoan-specific importin alpha gene family. J. Mol. Evol. 68: 351-365.

Mateo M, Reid SP, Leung LW, Basler CF, et al. (2010). Ebolavirus VP24 binding to karyopherins is required for inhibition of interferon signaling. J. Virol. 84: 1169-1175.

Mynott AV, Harrop SJ, Brown LJ, Breit SN, et al. (2011). Crystal structure of importin-alpha bound to a peptide bearing the nuclear localisation signal from chloride intracellular channel protein 4. FEBS J. 278: 1662-1675.

Pante $\mathrm{N}$ and Kann M (2002). Nuclear pore complex is able to transport macromolecules with diameters of about $39 \mathrm{~nm}$. Mol. Biol. Cell 13: 425-434.

Punta M, Coggill PC, Eberhardt RY, Mistry J, et al. (2012). The Pfam protein families database. Nucleic Acids Res. 40: D290-D301.

Ratan R, Mason DA, Sinnot B, Goldfarb DS, et al. (2008). Drosophila importin alpha1 performs paralog-specific functions essential for gametogenesis. Genetics 178: 839-850.

Sachan N, Mishra AK, Mutsuddi M and Mukherjee A (2013). The Drosophila importin-alpha3 is required for nuclear import of notch in vivo and it displays synergistic effects with notch receptor on cell proliferation. PLoS One 8: e68247.

Sasaki Y, Hagiwara K, Kakisaka M, Yamada K, et al. (2013). Importin alpha3/Qip1 is involved in multiplication of mutant influenza virus with alanine mutation at amino acid 9 independently of nuclear transport function. PLoS One 8: e55765.

Schaller T, Pollpeter D, Apolonia L, Goujon C, et al. (2014). Nuclear import of SAMHD1 is mediated by a classical karyopherin alpha/beta1 dependent pathway and confers sensitivity to VpxMAC induced ubiquitination and proteasomal degradation. Retrovirology 11: 29.

Tamura K, Peterson D, Peterson N, Stecher G, et al. (2011). MEGA5: Molecular evolutionary genetics analysis using maximum likelihood, evolutionary distance, and maximum parsimony methods. Mol. Biol. Evol. 28: 2731-2739.

Terry LJ, Shows EB and Wente SR (2007). Crossing the nuclear envelope: hierarchical regulation of nucleocytoplasmic transport. Science 318: 1412-1416.

Umegaki-Arao N, Tamai K, Nimura K, Serada S, et al. (2013). Karyopherin Alpha2 is essential for rRNA transcription and protein synthesis in proliferative keratinocytes. PLoS One 8: e76416.

Vetter IR, Arndt A, Kutay U, Gorlich D, et al. (1999). Structural view of the Ran-Importin beta interaction at 2.3 A resolution. Cell 97: 635-646.

Wang P, Palese P and O'Neill RE (1997). The NPI-1/NPI-3 (karyopherin alpha) binding site on the influenza a virus nucleoprotein NP is a nonconventional nuclear localization signal. J. Virol. 71: 1850-1856.

Wirthmueller L, Roth C, Banfield MJ and Wiermer M (2013). Hop-on hop-off: importin-alpha-guided tours to the nucleus in innate immune signaling. Front. Plant Sci. 4: 149.

Xia Q, Guo Y, Zhang Z, Li D, et al. (2009). Complete resequencing of 40 genomes reveals domestication events and genes in silkworm (Bombyx). Science 326: 433-436.

Xia Q, Zhou Z, Lu C, Cheng D, et al. (2004). A draft sequence for the genome of the domesticated silkworm (Bombyx mori). Science 306: 1937-1940.

Yang SNY, Takeda AAS, Fontes MRM, Harris JM, et al. (2010). Probing the specificity of binding to the major nuclear localization sequence-binding site of importin-alpha using oriented peptide library screening. J. Biol. Chem. 285: 19935-19946.

Young JC, Ly-Huynh JD, Lescesen H, Miyamoto Y, et al. (2013). The nuclear import factor importin alpha 4 can protect against oxidative stress. Biochim. Biophys. Acta Mol. Cell Res. 1833: 2348-2356.

Zhang W, Zhang C, Lv Z, Fang D, et al. (2012). Molecular characterization, tissue distribution, subcellular localization and actinsequestering function of a thymosin protein from silkworm. PLoS One 7: e31040.

Zhou W, Cao H, Yang X, Cong K, et al. (2013). Characterization of nuclear localization signal in the N terminus of integrin-linked kinase-associated phosphatase (ILKAP) and its essential role in the down-regulation of RSK2 protein signaling. J. Biol. Chem. 288: 6259-6271. 